\title{
Early Aspirin Discontinuation Following Acute Coronary Syndrome or Percutaneous Coronary Intervention: A Systematic Review and Meta-Analysis of Randomized Controlled Trials
}

\author{
Paul Guedeney ${ }^{1}$, Jules Mesnier ${ }^{1}$, Sabato Sorrentino ${ }^{2}$, Farouk Abcha ${ }^{1}{ }^{\oplus}$, Michel Zeitouni ${ }^{1}$, \\ Benoit Lattuca ${ }^{1}$, Johanne Silvain ${ }^{1}$, Salvatore De Rosa ${ }^{2} \mathbb{D}$, Ciro Indolfi ${ }^{2}{ }^{\mathbb{D}}$, Jean-Philippe Collet ${ }^{1}$, \\ Mathieu Kerneis ${ }^{1}$ and Gilles Montalescot ${ }^{1, *}$ \\ 1 Sorbonne University, ACTION Study Group, INSERM UMRS_1166, Cardiology Institute, Pitié Salpêtrière \\ Hospital (AP-HP) Paris, 75013 Paris, France; paul.guedeney@aphp.fr (P.G.); mesnier.jules@gmail.com (J.M.); \\ abchafarouk@gmail.com (F.A.); michel.zeitouni@gmail.com (M.Z.); \\ b-lattuca@chu-montpellier.mssante.fr (B.L.); johanne.silvain@aphp.fr (J.S.); \\ jean-philippe.collet@aphp.fr (J.-P.C.); mathieu.kerneis@aphp.fr (M.K.) \\ 2 Division of Cardiology, Department of Medical and Surgical Science, Magna Graecia University, \\ 88100 Catanzaro, Italy; sabatosorrentino@hotmail.com (S.S.); saderosa@unicz.it (S.D.R.); \\ indolfi@unicz.it (C.I.) \\ * Correspondence: gilles.montalescot@aphp.fr; Tel.: +33-142-1630-07
}

Received: 9 February 2020; Accepted: 28 February 2020; Published: 3 March 2020

\begin{abstract}
The respective ischemic and bleeding risks of early aspirin discontinuation following an acute coronary syndrome (ACS) or percutaneous coronary intervention (PCI) remain uncertain. We performed a prospero-registered review of randomized controlled trials (RCTs) comparing a P2 $\mathrm{Y}_{12}$ inhibitor-based single antiplatelet strategy following early aspirin discontinuation to a strategy of sustained dual antiplatelet therapy (DAPT) in ACS or PCI patients requiring, or not, anticoagulation for another indication (CRD42019139576). We estimated risk ratios (RR) and 95\% confidence intervals (CI) using random effect models. We included nine RCTs comprising 40,621 patients. Compared to prolonged DAPT, major bleeding ( $2.2 \%$ vs. $2.8 \%$; RR $0.68 ; 95 \%$ CI: 0.54 to $\left.0.87 ; p=0.002 ; \mathrm{I}^{2}: 63 \%\right)$, non-major bleeding (5.0 \% vs. $6.1 \%$; RR: $0.66 ; 95 \%$ CI: 0.47 to $0.94 ; p=0.02 ; I^{2}: 87 \%$ ) and all bleeding (7.4\% vs. $9.9 \%$; RR: $0.65 ; 95 \%$ CI: 0.53 to $0.79 ; p<0.0001 ; I^{2}: 88 \%$ ) were significantly reduced with early aspirin discontinuation without significant difference for all-cause death $(p=0.60)$, major adverse cardiac and cerebrovascular events (MACE) $(p=0.60)$, myocardial infarction (MI) $(p=0.77)$, definite stent thrombosis (ST) $(p=0.63)$, and any stroke $(p=0.59)$. In patients on DAPT after an ACS or a PCI, early aspirin discontinuation prevents bleeding events with no significant adverse effect on the ischemic risk or mortality.
\end{abstract}

Keywords: aspirin; $\mathrm{P} 2 \mathrm{Y}_{12}$ inhibitors; antiplatelet therapy; acute coronary syndrome; percutaneous coronary intervention

\section{Introduction}

The optimal antithrombotic regimen following ACS or PCI has known considerable evolutions over the last thirty years. However, current guidelines still recommend the continuation of prolonged DAPT including aspirin and a $\mathrm{P}_{2} \mathrm{Y}_{12}$ inhibitor based on ancient pivotal randomized trials [1-3]. Since then, implementation of newer generation drug-eluting stents (DES), the widespread use of lipid lowering therapy, and a new generation of $\mathrm{P}_{2} \mathrm{Y}_{12}$ inhibitors have led to a reduction of ST or non-stent 
related MI following PCI or ACS [4,5]. In these circumstances, the benefit of sustained DAPT may translate into a smaller absolute ischemic event risk reduction, which might be potentially outweighed by the associated higher risk of bleeding [6]. Since aspirin yields limited additional platelet inhibition when associated with $\mathrm{P}_{2} \mathrm{Y}_{12}$ inhibitors, aspirin-free strategies have been evaluated in several recent randomized controlled trials enrolling ACS or PCI patients; in some of these studies patients also had an indication for chronic oral anticoagulation (OAC) [7-19]. Most of these trials (but not all) reported lower rates of bleeding without aspirin, but all of them were underpowered as to properly evaluate the associated ischemic risk. This systematic review and meta-analysis aims to evaluate the safety and efficacy of early aspirin discontinuation with $\mathrm{P}_{2} \mathrm{Y}_{12}$ inhibitors single antiplatelet therapy continuation, as compared with a strategy of sustained DAPT following an ACS or PCI, in patients with or without concomitant OAC treatment.

\section{Materials and Methods}

\subsection{Research Strategy and Selection Criteria}

In accordance with the PRISMA (Preferred Reporting Items for Systematic Reviews and Meta-analysis) guidelines (Supplementary Table S1), we searched PubMed/Medline, CENTRAL (Cochrane Central Register of Controlled trials), clinicaltrials.gov, and slide presentations from the latest international conferences for relevant abstracts and manuscripts published up to 27 September, 2019. The following keywords were used: randomized controlled trial; acute coronary syndrome; percutaneous coronary intervention; antithrombotic therapy; aspirin; clopidogrel; ticagrelor; prasugrel; rivaroxaban; apixaban; edoxaban; dabigatran. Citations were screened at the title and abstract level and retrieved if considered relevant. The inclusion criterion was a RCT with a clinical primary endpoint, comparing a strategy of early aspirin discontinuation (i.e., with aspirin placebo or no aspirin treatment) and $\mathrm{P}_{2} \mathrm{Y}_{12}$ inhibitors (clopidogrel, ticagrelor, or prasugrel) continuation, to a strategy of prolonged DAPT including aspirin and $\mathrm{P}_{2} \mathrm{Y}_{12}$ inhibitors, following ACS or PCI, in patients with or without indication for chronic OAC. No restrictions on follow-up or study size were applied. The exclusion criteria were observational study design (including single-arm pilot studies), non-English-language studies, editorial, letters, expert opinions, case reports or series and studies with duplicated data. Two authors independently evaluated studies for eligibility and discrepancies were resolved by a third reviewer. The primary safety endpoint of interest was major bleeding, as defined in each trial (Supplementary Table S2). Other safety endpoints of interest were non-major bleeding as well as all bleeding, defined as the composite of major and non-major bleedings. The primary efficacy endpoint was all-cause death. Other efficacy endpoints of interest were MACCE, MI, definite ST, definite or probable ST, any stroke, and ischemic stroke as reported in each trial (Supplementary Table S3). The study is registered in PROSPERO (CRD42019139576).

\subsection{Data Extraction}

Relevant data elements, including baseline population and procedural characteristics, were independently collected from each trial into a pre-specified structural dataset. Efficacy and safety endpoints were collected at the longest available time of follow-up according to the intention-to-treat principle. Discrepancies in the data collection were resolved by consensus. The risk of bias of the included studies was assessed according to the Cochrane Collaboration guidelines. 


\subsection{Statistical Analysis}

Risk ratios and 95\% CI were estimated using Mantel-Haenszel random-effects models according to DerSimonian and Laird. Fixed effect models for all efficacy and safety endpoints were also reported in the Online documents. Heterogeneity among trials for each outcome was estimated with Chi-square tests and quantified with $\mathrm{I}^{2}$-statistics. Visual inspections of funnel plot were used to evaluate potential publication bias and small study effect. In order to evaluate the public health impact of the early aspirin discontinuation strategy on safety endpoints, we pooled outcomes data of all studies into a single population to calculate the absolute risk difference (ARD) and the number needed to treat (NNT) to avoid one bleeding event [20]. Sensitivity analyses were pre-specified: (i) evaluating the impact of safety events after exclusion of trials in which background OAC treatment was not homogenous between early aspirin discontinuation and prolonged DAPT groups; (ii) evaluating the safety of early aspirin discontinuation across various bleeding scales; (ii) evaluating the effect of early aspirin discontinuation according to the $\mathrm{P}_{2} \mathrm{Y}_{12}$ inhibitors predominantly used (i.e., clopidogrel or ticagrelor), and according to the delay before aspirin discontinuation (i.e., one month or three months) in trials without chronic indication for OAC; (iv) evaluating the effect of early aspirin discontinuation using adjudicated data from the GLOBAL LEADERS Adjudication Sub-Study (GLASSY) [21]. A $p$-value $<0.05$ was considered as statistically significant. Analyses were conducted using Cochrane's Review Manager (RevMan) version 5.3 (The Cochrane Collaboration, Copenhagen, Denmark).

\section{Results}

\subsection{Systematic Review}

A total of nine RCTs were included in the present meta-analysis (Supplementary Figure S1), comprising 40,621 patients of whom 20,320 (50\%) were treated with a strategy of early aspirin discontinuation. OAC treatment was present in five RCTs, as an inclusion criterion, representing 11,532 (28.4\%) patients. Main characteristics of the included trials are detailed in Table 1, baseline patients' characteristics are detailed in Table 2, and procedural characteristics are detailed in Supplementary Table S4. Male and diabetic patients represented $75.6 \%$ and $31.7 \%$ of the overall population respectively and the index event was an ACS in $52.4 \%$ of the cases. Median follow-up was 1 year (range 0.5 to 2 years).

\subsection{Safety Endpoints}

The association of early aspirin discontinuation with safety endpoints is detailed in Figure 1.

The early aspirin discontinuation strategy was associated with a significant reduction of major bleeding (2.2\% vs. $2.8 \%$; RR 0.68; 95\% CI: 0.54 to $0.87 ; p=0.002 ; \mathrm{I}^{2}: 63 \%$ ), with an ARD of $-0.62 \%$ and NNT: 162 ; non-major bleeding (5.0\% vs. $6.1 \%$; RR: $0.66 ; 95 \%$ CI: 0.47 to $0.94 ; p=0.02 ; I^{2}: 87 \%$ ), with an ARD of $-1.12 \%$ and NNT: 89 ; as well as all bleeding (7.4\% vs. $9.9 \%$; RR: $0.65 ; 95 \%$ CI: 0.53 to $0.79 ; p<$ $0.0001 ; \mathrm{I}^{2}: 88 \%$ ), with an ARD of $-2.57 \%$ and NNT: 39 . The effect of early aspirin discontinuation was consistent in patients with and without chronic background OAC, without significant interaction for major, non-major and all bleeding outcomes ( $p=0.78 ; p=0.31$ and $p=0.79$, respectively). 
Table 1. Characteristics of the included studies.

\begin{tabular}{|c|c|c|c|c|c|c|c|c|c|c|c|c|}
\hline \multirow[b]{2}{*}{$\begin{array}{c}\text { Study } \\
\text { Publication Year } \\
\text { Clinicaltrials.gov ID }\end{array}$} & \multirow[b]{2}{*}{ Study Design } & \multirow[b]{2}{*}{$\begin{array}{c}\text { Main } \\
\text { Inclusion } \\
\text { Criteria }\end{array}$} & \multirow[b]{2}{*}{$\begin{array}{l}\text { Main Exclusion } \\
\text { Criteria }\end{array}$} & \multirow[b]{2}{*}{$\begin{array}{c}\text { Sample } \\
\text { Size }\end{array}$} & \multirow[b]{2}{*}{ Follow Up } & \multicolumn{3}{|c|}{ Early Aspirin Discontinuation } & \multicolumn{3}{|c|}{ Standard of Care } & \multirow[b]{2}{*}{ Primary Outcomes } \\
\hline & & & & & & $\begin{array}{c}\text { Duration of } \\
\text { Aspirin Therapy } \\
\text { after } \\
\text { Randomization }\end{array}$ & $\begin{array}{l}\text { P2Y }_{12} \text { Inhibitors } \\
\text { Use (Dosage) }\end{array}$ & OAC Agent & $\begin{array}{c}\text { DAPT } \\
\text { Duration }\end{array}$ & $\begin{array}{l}\text { Antiplatelet } \\
\text { Agents }\end{array}$ & OAC Agent & \\
\hline $\begin{array}{c}\text { WOEST [11] } \\
2013 \\
\text { NCT00769938 }\end{array}$ & $\begin{array}{c}\text { Randomized, } \\
\text { open label, } \\
\text { multicentric, } \\
\text { superiority, } \\
\text { controlled trial } \\
\end{array}$ & $\begin{array}{l}\text { Indication for } \\
\text { oral } \\
\text { anticoagulation } \\
\text { and PCI }\end{array}$ & $\begin{array}{l}>80 \text { years, Prior } \\
\text { ICH, cardiogenic } \\
\text { shock recent } \\
\text { major bleeding, } \\
\text { thrombocytopenia }\end{array}$ & 563 & 12 months & $\begin{array}{c}\text { None after } \\
\text { randomization }\end{array}$ & $\begin{array}{l}\text { Clopidogrel 100\% } \\
\quad(75 \mathrm{mg})\end{array}$ & VKA & $\begin{array}{l}1 \text { to } 12 \\
\text { months }\end{array}$ & $\begin{array}{c}\text { Aspirin } \\
80-100 \mathrm{mg} \text {; and } \\
\text { Clopidogrel } \\
75 \mathrm{mg}\end{array}$ & VKA & $\begin{array}{c}\text { Safety: } \\
\text { Any episode of } \\
\text { bleeding (defined } \\
\text { by TIMI, GUSTO or } \\
\text { BARC classification }\end{array}$ \\
\hline $\begin{array}{c}\text { PIONEER } \\
\text { AF-PCI [12] } \\
2016 \\
\text { NCT01830543 }\end{array}$ & $\begin{array}{l}\text { Randomized, } \\
\text { open label, } \\
\text { multicentric, } \\
\text { controlled trial }\end{array}$ & $\begin{array}{l}\text { Non-valvular } \\
\text { AF PCI with } \\
\text { coronary stent } \\
\text { implantation }\end{array}$ & $\begin{array}{l}\text { Prior stroke/TIA, } \\
\text { recent GI } \\
\text { bleeding, severe } \\
\text { CKD, anemia } \\
\text { increase risk of } \\
\text { bleeding } \\
\text { contra-indication } \\
\text { for OAC } \\
\text { Active } \\
\text { malignancy }\end{array}$ & 2124 & 12 months & $\begin{array}{c}\text { None after } \\
\text { randomization }\end{array}$ & $\begin{array}{c}\text { Clopidogrel } \\
93.1 \%(75 \mathrm{mg}), \\
\text { Ticagrelor 5.2\% } \\
\text { (90 mg bid), } \\
\text { Prasugrel } 1.7 \% \\
(10 \mathrm{mg})\end{array}$ & $\begin{array}{c}\text { Rivaroxaban } \\
15 \mathrm{mg} \text { or } 10 \mathrm{mg}\end{array}$ & $\begin{array}{l}1,6 \text { or } 12 \\
\text { months }\end{array}$ & $\begin{array}{c}\text { Aspirin } \\
75-100 \mathrm{mg}, \\
\text { and Clopidogrel } \\
75 \mathrm{mg}, \text { Ticagrelor } \\
90 \mathrm{mg} \text { bid, } \\
\text { Prasugrel } 10 \mathrm{mg}\end{array}$ & $\begin{array}{c}\text { VKA } \\
\text { or } \\
\text { Rivaroxaban } \\
2.5 \mathrm{mg}\end{array}$ & $\begin{array}{c}\text { Safety: } \\
\text { Composite of: } \\
\text { Major and minor } \\
\text { TIMI bleeding and } \\
\text { bleeding requiring } \\
\text { medical attention }\end{array}$ \\
\hline $\begin{array}{c}\text { REDUAL-PCI [13] } \\
2017 \\
\text { NCT02164864 }\end{array}$ & $\begin{array}{l}\text { Phase IIIb, } \\
\text { randomized, } \\
\text { open label, } \\
\text { multicentric, } \\
\text { non-inferiority, } \\
\text { controlled trial }\end{array}$ & $\begin{array}{l}\text { Non valvular } \\
\text { AF Successful } \\
\text { PCI }<120 \mathrm{~h}\end{array}$ & $\begin{array}{c}\text { Prosthetic heart } \\
\text { valves, severe } \\
\text { CKD, recent } \\
\text { stroke, major } \\
\text { surgery or GI } \\
\text { bleeding }\end{array}$ & 2725 & 14 months * & $\begin{array}{c}\text { None after } \\
\text { randomization }\end{array}$ & $\begin{array}{c}\text { Clopidogrel } \\
86.6 \%(75 \mathrm{mg}), \\
\text { Ticagrelor 12.4\% } \\
\text { (90 mg bid) }\end{array}$ & $\begin{array}{l}\text { Dabigatran } 150 \\
\text { or } 110 \mathrm{mg} \text { bid }\end{array}$ & $\begin{array}{c}1 \text { month } \\
\text { (BMS) } \\
3 \text { months } \\
\text { (DES) }\end{array}$ & $\begin{array}{c}\text { Aspirin }<100 \mathrm{mg} \\
\text { and Clopidogrel } \\
75 \mathrm{mg}, \\
\text { Ticagrelor } 90 \mathrm{mg} \\
\text { bid }\end{array}$ & VKA & $\begin{array}{c}\text { Safety: } \\
\text { Time to event } \\
\text { analysis of first } \\
\text { major or clinically } \\
\text { relevant } \\
\text { non major ISTH } \\
\text { bleeding }\end{array}$ \\
\hline $\begin{array}{c}\text { GLOBAL } \\
\text { LEADERS [14] } \\
2018 \\
\text { NCT01813435 }\end{array}$ & $\begin{array}{l}\text { Randomized, } \\
\text { open label, } \\
\text { multicentric, } \\
\text { superiority, } \\
\text { controlled trial }\end{array}$ & $\begin{array}{c}\text { Clinical } \\
\text { indication of } \\
\text { PCI }\end{array}$ & $\begin{array}{l}\text { Need for OAC, } \\
\text { planned surgery, } \\
\text { recent stroke, } \\
\text { prior major } \\
\text { bleeding }\end{array}$ & 15,968 & 24 months & 30 days & $\begin{array}{l}\text { Ticagrelor 100\% } \\
\text { (90 mg bid) }\end{array}$ & N.A. & 12 months & $\begin{array}{c}\text { Aspirin } \\
\text { 75-100 mg, } \\
\text { and Clopidogrel } \\
75 \mathrm{mg} \text {, Ticagrelor } \\
90 \mathrm{mg} \text { bid }\end{array}$ & N.A. & $\begin{array}{c}\text { Efficacy: } \\
\text { Composite of } \\
\text { all-cause death or } \\
\text { non-fatal, new } \\
\text { Q-wave myocardial } \\
\text { infarction. }\end{array}$ \\
\hline $\begin{array}{c}\text { AUGUSTUS [15] } \\
\quad 2019 \\
\text { NCT02415400 }\end{array}$ & $\begin{array}{l}\text { Multicentric, } \\
\text { randomized } \\
\text { with two-two } \\
\text { factorial design, } \\
\text { double blinded, } \\
\text { non-inferiority, } \\
\text { controlled trial }\end{array}$ & $\begin{array}{l}\text { AF and recent } \\
\text { PCI or ACS } \\
\text { with planned } \\
\text { used of at } \\
\text { least } 6 \text { months } \\
\text { of } \mathrm{P}_{12}\end{array}$ & $\begin{array}{c}\text { Other indication } \\
\text { for OAC, severe } \\
\text { CKD, prior ICH, } \\
\text { coagulopathy, } \\
\text { planned CABG }\end{array}$ & 4614 & 6 months & $\begin{array}{c}\text { None after } \\
\text { randomization }\end{array}$ & $\begin{array}{c}\text { Clopidogrel } \\
93.2 \% \\
\text { (75 mg) } \\
\text { Ticagrelor } 5.9 \% \\
\text { (90 mg bid) } \\
\text { Prasugrel } 0.9 \% \\
\text { (10 mg) }\end{array}$ & $\begin{array}{l}\text { Apixaban } 5 \mathrm{mg} \\
\text { or } 2.5 \mathrm{mg} \text { bid } \\
\text { or VKA }\end{array}$ & 6 months & $\begin{array}{c}\text { Aspirin } 81 \mathrm{mg}, \\
\text { and Clopidogrel } \\
75 \mathrm{mg}, \text { Ticagrelor } \\
90 \mathrm{mg} \text { bid, } \\
\text { Prasugrel } 10 \mathrm{mg}\end{array}$ & $\begin{array}{c}\text { Apixaban } \\
5 \mathrm{mg} \text { or } \\
2.5 \mathrm{mg} \text { bid } \\
\text { or VKA }\end{array}$ & $\begin{array}{c}\text { Safety: } \\
\text { major or clinically } \\
\text { relevant non-major } \\
\text { ISTH bleeding }\end{array}$ \\
\hline
\end{tabular}


Table 1. Cont.

\begin{tabular}{|c|c|c|c|c|c|c|c|c|c|c|c|c|}
\hline \multirow[b]{2}{*}{$\begin{array}{c}\text { Study } \\
\text { Publication Year } \\
\text { Clinicaltrials.gov ID }\end{array}$} & \multirow[b]{2}{*}{ Study Design } & \multirow[b]{2}{*}{$\begin{array}{l}\text { Main } \\
\text { Inclusion } \\
\text { Criteria }\end{array}$} & \multirow[b]{2}{*}{$\begin{array}{l}\text { Main Exclusion } \\
\text { Criteria }\end{array}$} & \multirow[b]{2}{*}{$\begin{array}{l}\text { Sample } \\
\text { Size }\end{array}$} & \multirow[b]{2}{*}{ Follow Up } & \multicolumn{3}{|c|}{ Early Aspirin Discontinuation } & \multicolumn{3}{|c|}{ Standard of Care } & \multirow[b]{2}{*}{ Primary Outcomes } \\
\hline & & & & & & $\begin{array}{c}\text { Duration of } \\
\text { Aspirin Therapy } \\
\text { after } \\
\text { Randomization } \\
\end{array}$ & $\begin{array}{l}\text { P2Y }_{12} \text { Inhibitors } \\
\text { Use (Dosage) }\end{array}$ & OAC Agent & $\begin{array}{c}\text { DAPT } \\
\text { Duration }\end{array}$ & $\begin{array}{c}\text { Antiplatelet } \\
\text { Agents }\end{array}$ & OAC Agent & \\
\hline $\begin{array}{c}\text { STOPDAPT-2 [16] } \\
2019 \\
\text { NCT02619760 }\end{array}$ & $\begin{array}{l}\text { Randomized, } \\
\text { open label, } \\
\text { multicentric, } \\
\text { non inferiority, } \\
\text { controlled trial }\end{array}$ & $\begin{array}{l}\text { PCI with } \\
\text { CoCr-EES } \\
\text { without } \\
\text { periprocedural } \\
\text { complication }\end{array}$ & $\begin{array}{l}\text { Need for OAC, } \\
\text { prior ICH, use of } \\
\text { other stents }\end{array}$ & 3009 & 12 months & 1 month & $\begin{array}{c}\text { During 1st month } \\
\text { Clopidogrel } \\
60.2 \%(75 \mathrm{mg}) \\
\text { Prasugrel } 39.6 \% \\
\text { (10 mg) } \\
\text { After 1st month } \\
\text { Clopidogrel 100\% } \\
(75 \mathrm{mg})\end{array}$ & N.A. & 12 months & $\begin{array}{l}\text { Aspirin } 81 \text { to } \\
200 \mathrm{mg} \text { and } \\
\text { Clopidogrel } \\
75 \mathrm{mg} \text { or } \\
\text { Prasugrel } 10 \mathrm{mg} \\
\text { before } 1 \mathrm{month}, \\
\text { Followed by } \\
\text { Clopidogrel } \\
75 \mathrm{mg}\end{array}$ & N.A. & $\begin{array}{l}\text { Safety and efficacy: } \\
\text { Composite of } \\
\text { cardiovascular } \\
\text { death, MI, definite } \\
\text { stent thrombosis, } \\
\text { stroke and TIMI } \\
\text { major and minor } \\
\text { bleeding }\end{array}$ \\
\hline $\begin{array}{c}\text { SMART } \\
\text { CHOICE [17] } \\
2019 \\
\text { NCT02079194 }\end{array}$ & $\begin{array}{l}\text { Randomized, } \\
\text { open label, } \\
\text { multicentric, } \\
\text { non inferiority, } \\
\text { controlled trial }\end{array}$ & $\begin{array}{l}\text { PCI with DES } \\
\text { for ACS or } \\
\text { stable CAD }\end{array}$ & $\begin{array}{l}\text { Hemodynamic } \\
\text { instability; active } \\
\text { bleeding; recent } \\
\text { DES implantation }\end{array}$ & 2993 & 12 months & 3 months & $\begin{array}{c}\text { Clopidogrel } \\
77.9 \% \\
(75 \mathrm{mg}) \\
\text { Ticagrelor 6.5\% } \\
(90 \mathrm{mg} \text { bid) } \\
\text { Prasugrel } 0.7 \% \\
\quad(10 \mathrm{mg}) \\
\end{array}$ & $\begin{array}{l}\text { investigators } \\
\text { choice }\end{array}$ & 12 months & $\begin{array}{c}\text { Clopidogrel } \\
75 \text { mg, Ticagrelor } \\
90 \mathrm{mg} \text { bid, } \\
\text { Prasugrel } 10 \mathrm{mg}\end{array}$ & $\begin{array}{l}\text { investigators } \\
\text { choice }\end{array}$ & $\begin{array}{c}\text { Efficacy: } \\
\text { Composite of } \\
\text { all-cause mortality, } \\
\text { MI, stroke }\end{array}$ \\
\hline $\begin{array}{l}\text { ENTRUST-AF } \\
\text { PCI [18] } \\
2019 \\
\text { NCT02866175 }\end{array}$ & $\begin{array}{l}\text { Phase IIIb, } \\
\text { Randomized, } \\
\text { open label, } \\
\text { multicentric, } \\
\text { controlled trial }\end{array}$ & $\begin{array}{l}\text { Non valvular } \\
\text { AF and PCI } \\
\text { procedure for } \\
\text { stable CAD or } \\
\text { ACS with } \\
\text { success }\end{array}$ & $\begin{array}{l}\text { Valvular or } \\
\text { reversible AF, } \\
\text { mechanical heart } \\
\text { valve, severe } \\
\text { CKD, major } \\
\text { surgery planned, } \\
\text { recent ischemic } \\
\text { stroke, high } \\
\text { bleeding risk }\end{array}$ & 1506 & 12 months & $\begin{array}{c}\text { None after } \\
\text { randomization }\end{array}$ & $\begin{array}{l}\text { Clopidogrel } \\
92.7 \% \\
(75 \mathrm{mg}) \\
\text { Ticagrelor } 6.5 \% \\
\text { (90 mg bid) } \\
\text { Prasugrel } 0.7 \% \\
\text { (5 or } 10 \mathrm{mg})\end{array}$ & $\begin{array}{c}\text { Edoxaban } \\
60 \mathrm{mg} \text { or } 30 \mathrm{mg} \\
\text { VKA }\end{array}$ & $\begin{array}{l}1 \text { to } 12 \\
\text { months }\end{array}$ & $\begin{array}{c}\text { Aspirin } 100 \mathrm{mg}, \\
\text { and Clopidogrel } \\
75 \mathrm{mg} \text {, Ticagrelor } \\
90 \mathrm{mg} \text { bid, } \\
\text { Prasugrel } 5 \text { or } \\
10 \mathrm{mg}\end{array}$ & VKA & $\begin{array}{c}\text { Safety: } \\
\text { Composite of ISTH } \\
\text { major and clinically } \\
\text { relevant non-major } \\
\text { bleeding } \\
\text { Efficacy: } \\
\text { Composite of CV } \\
\text { death, stroke, } \\
\text { systemic embolic } \\
\text { event, MI and } \\
\text { definite ST }\end{array}$ \\
\hline $\begin{array}{c}\text { TWILIGHT [19] } \\
2019 \\
\text { NCT02270242 }\end{array}$ & $\begin{array}{l}\text { Phase IV, } \\
\text { randomized, } \\
\text { blinded-label, } \\
\text { multicentric, } \\
\text { superiority } \\
\text { controlled trial }\end{array}$ & $\begin{array}{l}\text { High risk } \\
\text { patients with } \\
\text { complex } \\
\text { PCI } †\end{array}$ & $\begin{array}{l}\text { Contraindication } \\
\text { to aspirin or } \\
\text { ticagrelor, STEMI } \\
\text { as index event, } \\
\text { need for chronic } \\
\text { OAC, prior } \\
\text { stroke, planned } \\
\text { surgery or } \\
\text { coronary } \\
\text { revascularization }\end{array}$ & 7119 & 12 months & $\begin{array}{l}\text { None after } \\
\text { randomization }\end{array}$ & Ticagrelor $(100 \%)$ & N.A. & 12 months & $\begin{array}{l}\text { Aspirin } \\
81-100 \mathrm{mg} \\
\text { and Ticagrelor } \\
90 \mathrm{mg} \text { bid }\end{array}$ & N.A. & $\begin{array}{c}\text { Safety: } \\
\text { Composite of } \\
\text { BARC types 2, } 3 \text { or } \\
5 \text { bleeding }\end{array}$ \\
\hline
\end{tabular}

* mean follow-up + was defined as the association of at least one criterion among: age $>65$ years, female sex, established CV disease, diabetes mellitus, chronic kidney disease, and at least one criterion among: multivessel disease, total stent length $>30 \mathrm{~mm}$, thrombotic lesion, bifurcation, left main or proximal left anterior descending artery. PCI: percutaneous coronary intervention; DAPT: Dual antiplatelet therapy; OAC: oral anticoagulation; VKA: vitamin K antagonist; MI: myocardial infarction; AF: atrial fibrillation; TIA: transient ischemic attack; ICH: intracranial hemorrhage; GI: gastro-intestinal; CKD: Chronic kidney disease; ACS: acute coronary syndrome; CABG: coronary artery bypass graft; CAD: coronary artery disease; CV: cardiovascular; CoCr-EES: cobalt-chromium everolimus eluting stent; N.A.: not applicable; STEMI: ST segment elevation myocardial infarction. 
Table 2. Baseline patients' characteristics.

\begin{tabular}{|c|c|c|c|c|c|c|c|c|c|c|}
\hline Study & Male Sex & Age (Years) * & Prior MI & $\begin{array}{c}\text { Prior Coronary } \\
\text { Revascularization }\end{array}$ & $\begin{array}{l}\text { Diabetes } \\
\text { Mellitus }\end{array}$ & $\begin{array}{c}\text { Systemic } \\
\text { Hypertension }\end{array}$ & Dyslipidemia & $\begin{array}{c}\text { Active } \\
\text { Smoking }\end{array}$ & $\begin{array}{c}\text { ACS as } \\
\text { ildex Rvent }\end{array}$ & Type of Stent Used \\
\hline WOEST & $448(79.6 \%)$ & $\begin{array}{l}\text { EAD: } 70.3 \pm 7.0 \\
\text { DAPT: } 69.5 \pm 8.0\end{array}$ & $196(34.8 \%)$ & $\begin{array}{l}\text { PCI: } 187(33.2 \%) \\
\text { CABG: } 130(23.1 \%)\end{array}$ & $140(24.9 \%)$ & $386(68.6 \%)$ & $396(70.3 \%)$ & $102(18.1 \%)$ & $155(27.5 \%)$ & $\begin{array}{c}\text { None: } 9(1.6 \%) \\
\text { DES: } 364(64.6 \%) \\
\text { BMS: } 175(31.1 \%) \\
\text { Both: } 14(2.5 \%)\end{array}$ \\
\hline $\begin{array}{l}\text { PIONEER } \\
\text { AF-PCI }\end{array}$ & $1581(74.4 \%)$ & $\begin{array}{c}\text { EAD: } 70.4 \pm 9.1 \\
\text { DAPT: } 70.0 \pm 9.1 \text { and } \\
69.9 \pm 8.7\end{array}$ & $477(22.5 \%)$ & - & $624(29.4 \%)$ & $1571(74.0 \%)$ & $913(43.0 \%)$ & $141(6.6 \%)$ & $1096(51.6 \%)$ & $\begin{array}{l}\text { DES: } 1403(66.0 \%) \\
\text { BMS: } 675(31.8 \%) \\
\text { Both: } 40(1.9 \%)\end{array}$ \\
\hline REDUAL-PCI & $2070(76.0 \%)$ & $\begin{array}{c}\text { EAD: } 71.5 \pm 8.9 \text { and } \\
68.6 \pm 7.7 \\
\text { DAPT: } 71.7 \pm 8.9\end{array}$ & $699(25.6 \%)$ & $\begin{array}{c}\text { PCI: } 912(33.5 \%) \\
\text { CABG: } 287(10.5 \%)\end{array}$ & $993(36.4 \%)$ & - & - & - & $1375(50.5 \%)$ & $\begin{array}{c}\text { DES: } 2251(82.8 \%) \\
\text { BMS: } 404(14.9 \%) \\
\text { Both: } 41(1.5 \%) \\
\text { Other: } 21(0.8 \%) \\
\end{array}$ \\
\hline $\begin{array}{l}\text { GLOBAL } \\
\text { LEADERS }\end{array}$ & $\begin{array}{c}12,254 \\
(76.7 \%)\end{array}$ & $\begin{array}{l}\text { EAD: } 64.5 \pm 10.3 \\
\text { DAPT: } 64.6 \pm 10.3\end{array}$ & $3710(23.2 \%)$ & $\begin{array}{l}\text { PCI: 5,221 (32.7\%) } \\
\text { CABG: } 943(5.9 \%)\end{array}$ & $4038(25.3 \%)$ & $11,715(73.4 \%)$ & $10,768(67.4 \%)$ & $4169(26.1 \%)$ & $7487(46.9 \%)$ & $\begin{array}{c}\text { Biolimus A9-eluting } \\
\text { stent: } 94.6 \% \text { of lesions; } \\
\text { other stent in } 6.5 \% \text { of } \\
\text { lesions }\end{array}$ \\
\hline AUGUSTUS & $3277(71.0 \%)$ & $\begin{array}{l}\text { EAD: } 70.8(64.4-77.3) \\
\text { DAPT: } 70.6(63.8-77.2)\end{array}$ & - & - & $1678(36.4 \%)$ & $4073(88.3 \%)$ & - & - & $2811(60.2 \%)$ & - \\
\hline STOPDAPT-2 & $2337(77.7 \%)$ & $\begin{array}{l}\text { EAD: } 68.1 \pm 10.9 \\
\text { DAPT: } 69.1 \pm 10.4\end{array}$ & $406(13.5 \%)$ & $\begin{array}{l}\text { PCI: } 1032(34.3 \%) \\
\text { CABG: } 59(2.0 \%)\end{array}$ & $1159(38.5 \%)$ & $2221(73.8 \%)$ & $2244(74.6 \%)$ & $710(23.6 \%)$ & $1148(38.2 \%)$ & CoCr-EES \\
\hline $\begin{array}{l}\text { SMART } \\
\text { CHOICE }\end{array}$ & $2198(73.4 \%)$ & $\begin{array}{l}\text { EAD: } 64.6 \pm 10.7 \\
\text { DAPT: } 64.4 \pm 10.7\end{array}$ & $127(4.2 \%)$ & $349(11.7 \%)$ & $1122(37.5 \%)$ & $1840(61.5 \%)$ & $1352(45.2 \%)$ & $791(26.4 \%)$ & $1741(58.2 \%)$ & $\begin{array}{c}\text { CoCr-EES: } 1051(35.1 \%) \\
\text { PtCr-EES: } 967(32.3 \%) \\
\text { BP-SES: } 972(32.5 \%)\end{array}$ \\
\hline $\begin{array}{l}\text { ENTRUST-AF } \\
\text { PCI }\end{array}$ & $1120(74.4 \%)$ & $\begin{array}{l}\text { EAD: } 69 \text { (63-77) } \\
\text { DAPT: } 70(64-77)\end{array}$ & $365(24.2 \%)$ & $\begin{array}{l}\text { PCI: } 394(26.2 \%) \\
\text { CABG: } 95(6.3 \%)\end{array}$ & $517(34.3 \%)$ & $1361(90.4 \%)$ & $981(65.1 \%)$ & - & 777 (51.6 \%) & - \\
\hline TWILIGHT & $5421(76.1 \%)$ & $\begin{array}{l}\text { EAD: } 65.2 \pm 10.3 \\
\text { DAPT: } 65.1 \pm 10.4\end{array}$ & $2040(28.7 \%)$ & $\begin{array}{l}\text { PCI: } 2998(42.1 \%) \\
\text { CABG: } 710(10.0 \%)\end{array}$ & $2620(36.8 \%)$ & $5154(72.4 \%)$ & $4303(60.4 \%)$ & $1548(21.8 \%)$ & $4614(64.8 \%)$ & Locally approved DES \\
\hline
\end{tabular}

${ }^{*}$ Age is provided as mean \pm standard deviation or as median [IQR]; MI: myocardial infarction; ACS: acute coronary syndrome; DES: drug eluting stent; BMS: bare metal stent; STEMI: ST segment elevation myocardial infarction; NSTEMI: non-ST segment elevation myocardial infarction; CoCr-EES: Cobalt-chromium everolimus eluting stent; PtCr-EES: Platinum-chromium everolimus eluting stent; BP-SES: Sirolimus-eluting stent with biodegradable polymer; PCI: percutaneous coronary intervention; CABG: coronary artery bypass graft; EAD: early aspirin discontinuation group; DAPT: dual antiplatelet therapy group. 


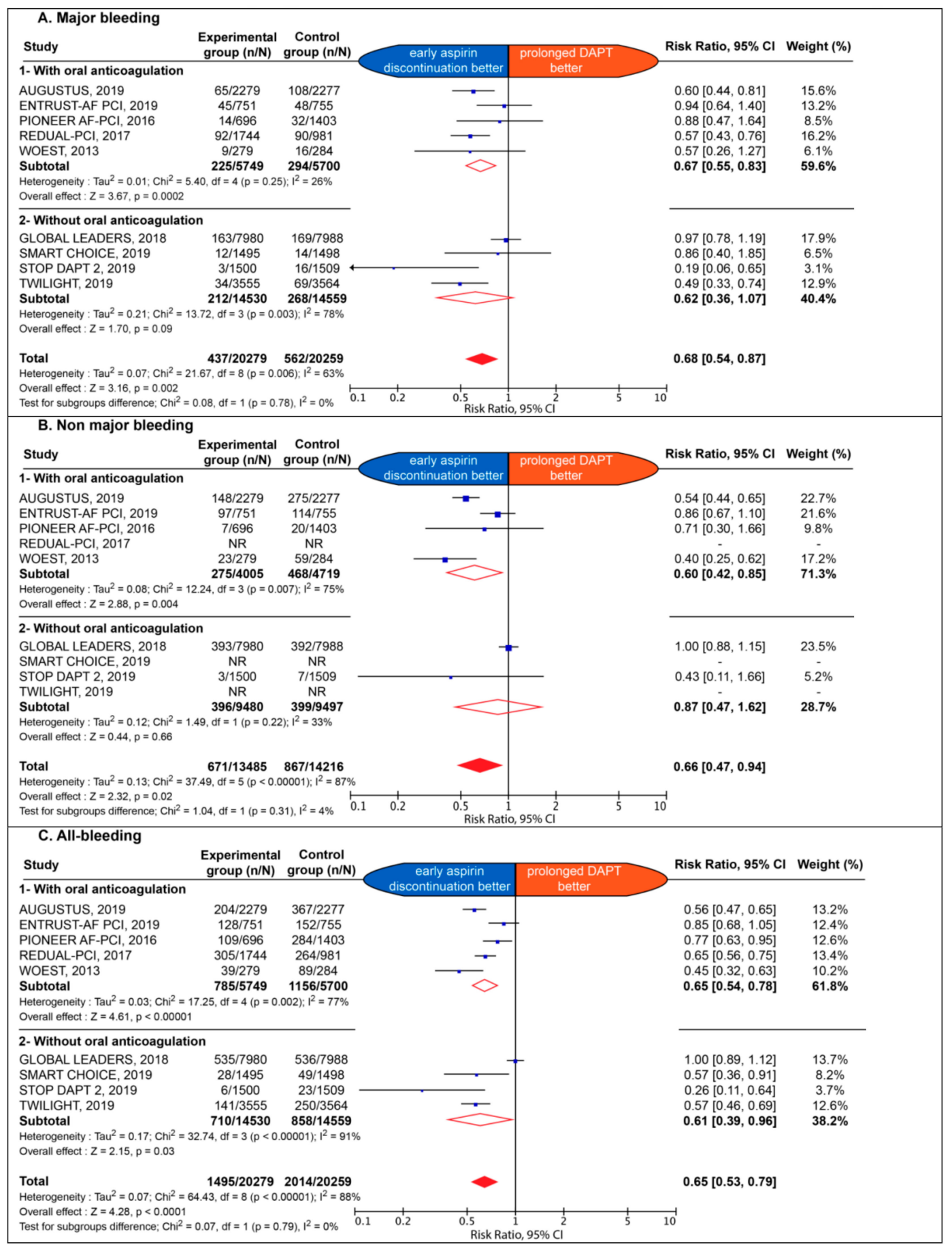

Figure 1. Estimate risk of major bleeding (A), non-major bleeding (B), and all bleeding (C). CI: confidence interval; DAPT: dual antiplatelet therapy; NR: not reported.

\subsection{Efficacy Endpoints}

The early impact of early aspirin discontinuation vs. prolonged DAPT on efficacy endpoint is detailed in Figures 2 and 3. 


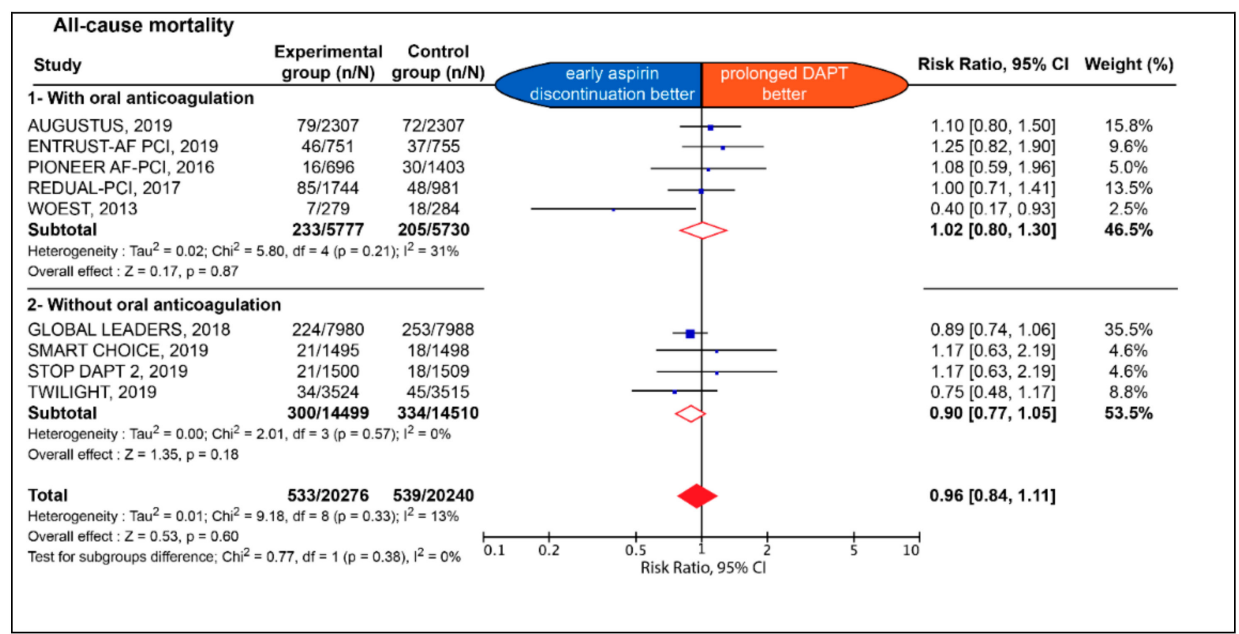

Figure 2. Estimate risk of all-cause death. CI: confidence interval; DAPT: dual antiplatelet therapy.

No significant difference between the two strategies was observed with respect to all-cause death (2.6\% vs. $2.7 \%$; RR: 0.96 ; $95 \%$ CI: 0.84 to $1.11 ; p=0.60 ; I^{2}: 13 \%$ ), MACCE (5.4\% vs. $5.3 \%$; RR: 0.97 ; 95\% CI 0.87 to $1.08 ; p=0.60$; I $\left.{ }^{2}: 28 \%\right)$, MI (2.0\% vs. $2.0 \%$; RR: $1.02 ; 95 \%$ CI: 0.88 to $1.19 ; p=0.77$; $\mathrm{I}^{2}: 8 \%$ ), definite stent thrombosis ( $0.79 \%$ vs. $0.71 \%$; RR: $1.07 ; 95 \%$ CI: 0.81 to $\left.1.43 ; p=0.63 ; \mathrm{I}^{2}: 0 \%\right)$, definite or probable stent thrombosis $\left(0.48 \%\right.$ vs. $0.37 \%$; RR: $1.34 ; 95 \%$ CI: 0.68 to $2.62 ; p=0.40$; I ${ }^{2}$ : $37 \%$ ) (Supplementary Figure S2), any stroke (0.99\% vs. $1.03 \%$; RR: $0.94 ; 95 \%$ CI: 0.76 to $1.17 ; p=0.59$; $\left.\mathrm{I}^{2}: 0 \%\right)$, as well as ischemic stroke (0.69\% vs. $0.73 \%$ RR: $0.97 ; 95 \%$ CI: $\left.0.61-1.53 ; p=0.89 ; \mathrm{I}^{2}: 44 \%\right)$ (Supplementary Figure S3). The effect of early aspirin discontinuation was consistent in patients with and without chronic background $\mathrm{OAC}$, without any significant interaction for all-cause death, MACCE, definite stent thrombosis, definite or probable stent thrombosis, any stroke, and ischemic stroke ( $p=0.38 ; p=0.27 ; p=0.55 ; p=0.28 ; p=0.87$ and $p=0.85$, respectively). There was a significant interaction between patients with and without chronic background OAC for MI $(p=0.04)$.

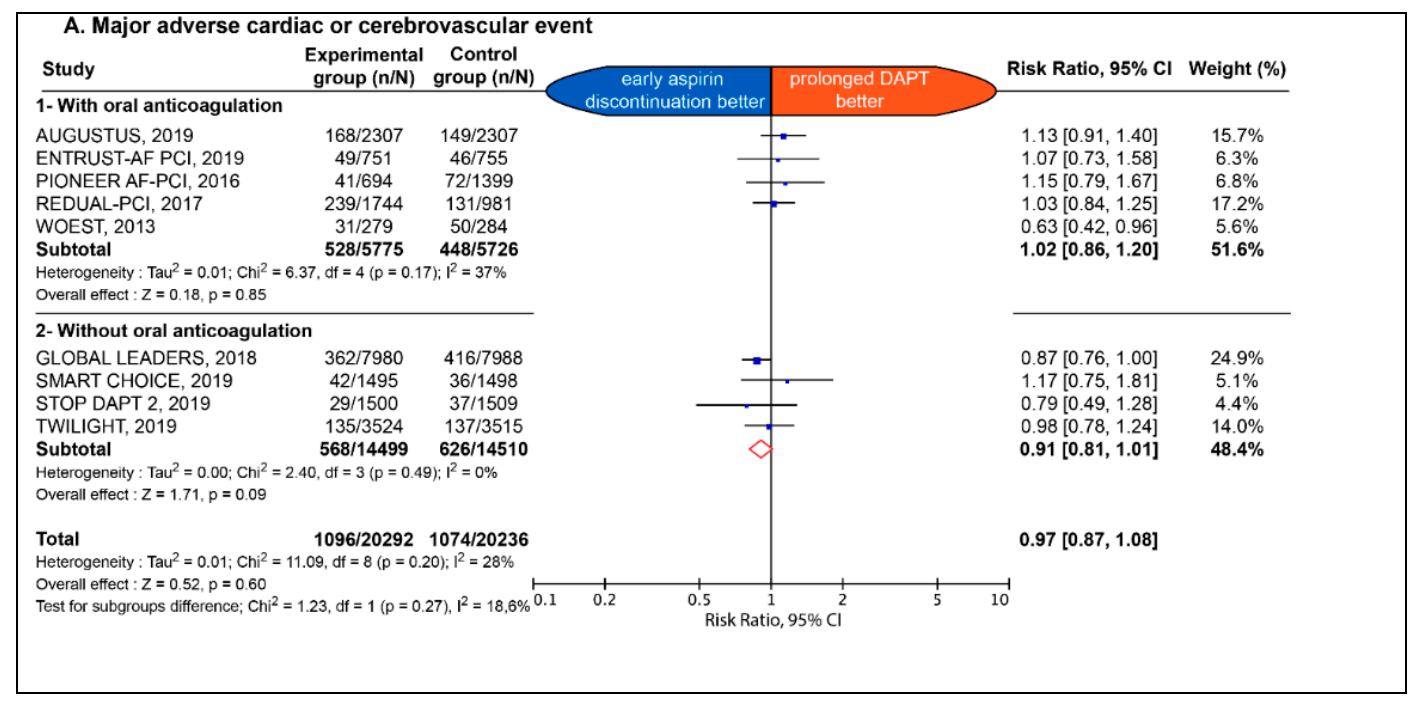

Figure 3. Cont. 


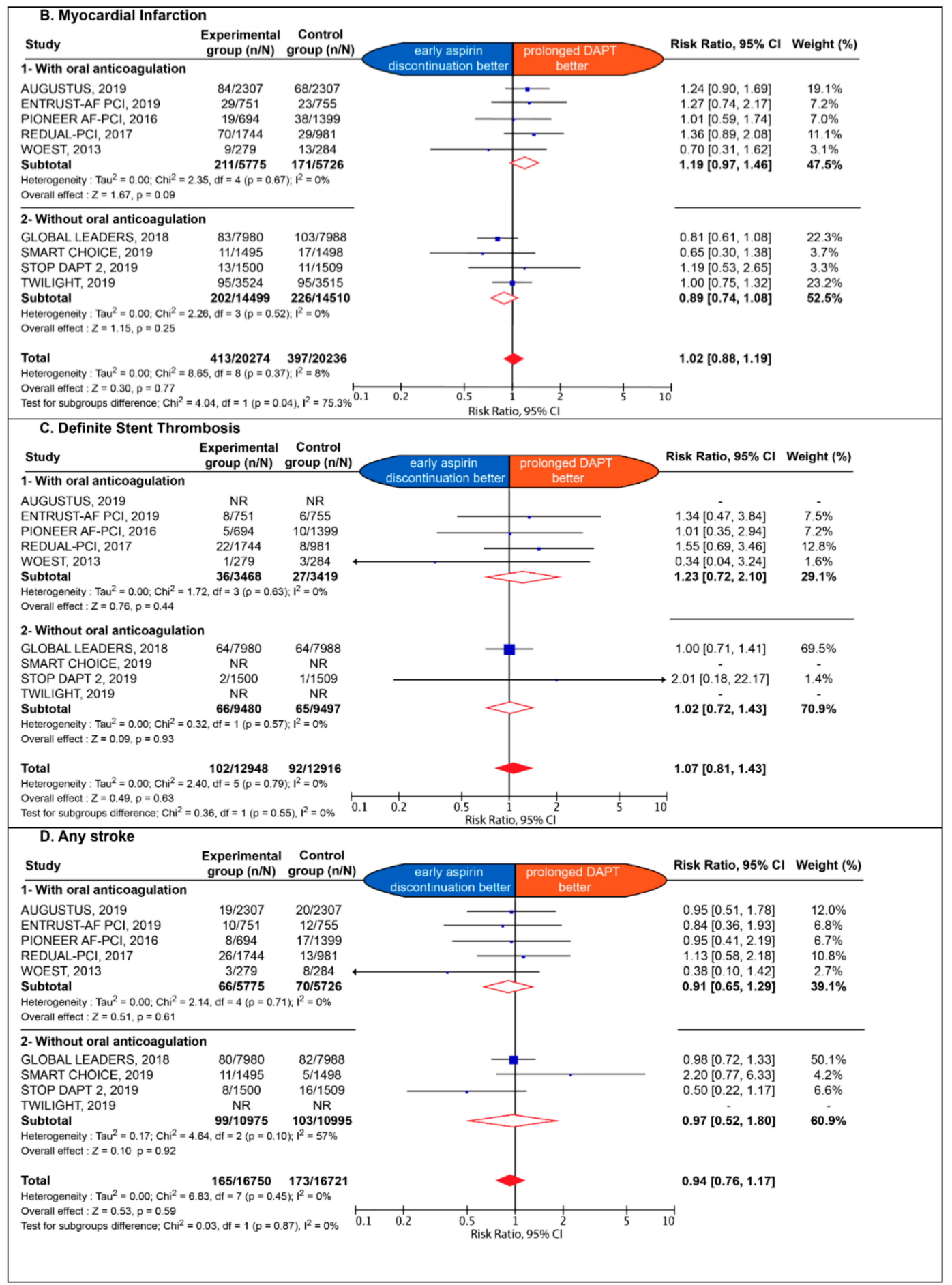

Figure 3. Estimate risk of major adverse cardiac and cerebrovascular events (A), myocardial infarction (B), definite stent thrombosis (C) and any stroke (D). CI: confidence interval; DAPT: dual antiplatelet therapy; NR: not reported.

\subsection{Sensitivity Analyses and Bias Assessment}

Results for the safety and efficacy endpoints remained consistent with the application of a fixed effect model (Supplementary Figure S4 and Supplementary Figure S5, respectively). The results of early aspirin discontinuation on safety events remained consistent after exclusion of RCT without homogenous background OAC between the experimental and control groups (Supplementary Figure S6). The association of early aspirin discontinuation with major, non-major, and major 
or non-major bleeding remained consistent across the various bleeding scales used in each trial (Supplementary Figures S7-S10). The results of early aspirin discontinuation on safety and efficacy events remained consistent according to the type of $\mathrm{P}^{2} \mathrm{Y}_{12}$ inhibitors predominantly used (i.e., clopidogrel vs. ticagrelor) (Supplementary Figure S11) or the duration of DAPT prior to aspirin discontinuation (i.e., 1 month vs. 3 month DAPT duration) (Supplementary Figure S12), in trials where patients had no indication for chronic OAC. Finally, the interaction between patients with or without OAC with respect to the association of early aspirin discontinuation with MI remained significant when using adjudicated MI from the GLOBAL LEADERS Adjudicated Sub-Study (GLASSY) trial (Supplementary Figure S13), but was no longer significant when using site-reported MI (Supplementary Figure S14). No evidence of publication bias or small study effect was found for both safety and efficacy outcomes (Supplementary Figure S15). Internal bias assessment for each study is reported in Supplementary Table S5.

\section{Discussion}

The choice, at the individual level, of the optimal antiplatelet strategy following ACS or PCI is a conundrum that requires stratifying both ischemic and bleeding risks. Our analysis, based on very recent randomized trials, comprising a total of 40,621 patients, demonstrates that early discontinuation of aspirin following ACS or PCI in patients with or without concomitant OAC treatment is associated with a significant reduction of major, non-major and all bleedings (Graphical abstract). This improved safety is not associated with any significant difference of all-cause death, MACCE, MI, definite ST, definite or probable ST, any stroke, or ischemic stroke.

Historically, aspirin is the first line antithrombotic treatment in cardiovascular diseases [8]. Subsequently, novel antiplatelet agents, including P2 $\mathrm{Y}_{12}$ inhibitors, were evaluated on top of aspirin, in pivotal RCTs $[9,22]$. Various DAPT regimens, based on aspirin with more or less prolonged duration of more or less potent $\mathrm{P}_{2} \mathrm{Y}_{12}$ inhibitors, have been evaluated to further reduce the ischemic residual risk, following ACS or PCI [23-25]. However, any reduction of the thrombotic risk has usually been offset by an increased risk of bleeding [22,24]. Of importance, bleeding following PCI has been associated with all-cause mortality and is thus paramount to prevent [26].

In particular, patients with AF requiring oral anticoagulants, presenting with an ACS or undergoing $\mathrm{PCI}$, are exposed to a considerable increased risk of bleeding. Recent large RCTs and meta-analyses have demonstrated that a dual therapy based on a non-vitamin $\mathrm{K}$ oral anticoagulant and a $\mathrm{P} \mathrm{Y}_{12}$ inhibitor is superior to triple therapy based on VKA with DAPT to prevent bleeding $[12,13,15,18,27,28]$. However, a number of these trials also reported an increase, albeit not significant, of coronary ischemic events in case of aspirin discontinuation [13,15]. In the present meta-analysis, rates of MI or definite ST were also numerically higher in patients treated with background OAC and early discontinuation of aspirin, but did not reach statistical significance, despite substantially increased statistical power. In patients undergoing PCI, short DAPT regimens have been associated with a higher risk of stent thrombosis. However, this effect was mainly observed with first-generation DES and was no longer observed with second-generation DES, which was overwhelmingly used in the studies included in the present meta-analysis [6].

In our study, clopidogrel and ticagrelor were predominantly used as $\mathrm{P}^{2} \mathrm{Y}_{12}$ inhibitors based single antiplatelet therapy. Considering the significant proportion of patients presenting with inadequate response to clopidogrel therapy, as detected by platelet function or genetic testing, concerns were raised regarding its use as single antiplatelet therapy, particularly in patients without background OAC [29]. However, genotype or platelet function test-based strategies of $\mathrm{P}_{2} \mathrm{Y}_{12}$ inhibitors have not resulted in significant reduction of ischemic complications in dedicated trials, which further suggests that clopidogrel alone may be safely used in these patients [30]. Consistently, no significant interaction was found between the effect of early aspirin discontinuation and prolonged clopidogrel or ticagrelor single antiplatelet therapy in patients not requiring chronic OAC in our study. Recently, the Intracoronary Stenting and Antithrombotic Regimen: Rapid Early Action for Coronary Treatment (ISAR-REACT) 5 
trial reported the superiority of prasugrel compared to ticagrelor in patients presenting with ACS for whom invasive evaluation is planned [31]. Prasugrel was only used in a minority of patients in the trials included in our meta-analysis and no conclusion can thus be drawn regarding its use as single antiplatelet therapy although, prasugrel has been used as single therapy in a few prior studies [32]. Of note, ticagrelor and not prasugrel is being evaluated as $\mathrm{P}_{2} \mathrm{Y}_{12}$ inhibitor based single antiplatelet therapy in other ongoing RCTs (ISRCTN84335288, NCT03447379, NCT03797651, and NCT02494895). Future trials are warranted to compare the performance of all commercially available $\mathrm{P}_{2} \mathrm{Y}_{12}$ inhibitors, used in clinical practice in the setting of early aspirin discontinuation.

Moreover, the optimal timing for aspirin discontinuation remains unclear. In all trials with patients presenting an underlying indication for chronic $\mathrm{OAC}$, aspirin use was authorized during $\mathrm{PCI}$ and prior to randomization which usually occurred between four hours after arterial sheath removal up to 14 days after PCI/ACS [11-13,15,18]. Conversely, in trials enrolling patients without indication for chronic $\mathrm{OAC}$, aspirin discontinuations occurred either at one month or at three months after randomization (mean weighted DAPT duration at 1.7 months). Of note, we did not find any significant interaction in the effect of aspirin discontinuation between one month and three months.

We report a significant interaction between background OAC and the risk of MI associated with early aspirin discontinuation. Although the role of pure chance cannot be excluded, as well as an issue with MI definitions as suggested by the sensitivity analysis, this effect might be real, reflecting the higher risk of OAC-treated patients, who are usually older and frailer than those without an indication for OAC.

\section{Limitations}

Several limitations are to be acknowledged. Firstly, our findings are subject to the inherent limitations of the included RCTs, subsequent to the study design, follow-up, ischemic and bleeding events definitions, and events ascertainment. This is particularly the case for GLOBAL LEADERS where all events but new Q-wave MI were site-reported. Secondly, as we lacked patient-level data, we were unable to perform time-to-event analysis or to evaluate the safety and efficacy of the early aspirin discontinuation strategy according to clinical and procedural complexity. Thirdly, we included studies with heterogeneous inclusion/exclusion criteria, particularly regarding the underlying indication for chronic $\mathrm{OAC}$, as well as differences in duration of antithrombotic treatment (i.e., overall duration for aspirin, $\mathrm{P}_{2} \mathrm{Y}_{12}$ inhibitors, or oral anticoagulation) which led to some degree of heterogeneity in the results. Fourthly, the risk of stent thrombosis decreases over time with new generation DES while we used a random effect modeling which assumes an equal chance of event at all time.

\section{Conclusions}

In patients on DAPT for an ACS or after a PCI, with or without an underlying indication for chronic OAC, a strategy of early aspirin discontinuation is associated with a significant reduction of major, non-major, and all bleeding, without detectable impact on mortality or ischemic risk.

Supplementary Materials: The following are available online at http://www.mdpi.com/2077-0383/9/3/680/s1: Figure S1. Flow diagram of the study selection process; Figure S2. Estimated risk of definite or probable stent thrombosis; Figure S3. Estimated risk of ischemic stroke; Figure S4. Estimated risk of safety event according to fixed effect model; Figure S5. Estimated risk of efficacy event according to fixed effect model; Figure S6. Estimated risk of safety events after exclusion of RCTs without homogenous background OAC between the compared groups; Figure S7. Estimated risk of safety event according to the BARC classification; Figure S8. Estimated risk of safety event according to the GUSTO classification; Figure S9. Estimated risk of safety event according to the ISTH classification; Figure S10. Estimated risk of safety event according to the TIMI classification; Figure S11. Estimated risk of safety event (A) and efficacy events (B) according to the $\mathrm{P}_{2} \mathrm{Y}_{12}$ inhibitors predominantly used in trials without indication for chronic oral anticoagulation; Figure S12. Estimated risk of safety event (A) and efficacy events (B) according to the DAPT duration prior to aspirin discontinuation in trials without indication for chronic oral anticoagulation; Figure S13. Estimated risk of efficacy (A) and safety (B) events using adjudicated data from GLASSY trial; Figure S14. Estimated risk of myocardial infarction using site-reported events with the GLOBAL LEADERS trial; Figure S15. Funnel plots and Table S1. PRISMA checklist items; Table S2. Definitions of major, 
non-major, and all bleeding used in each trial; Table S3. Definitions of major adverse cardiac and cerebrovascular events in each trial; Table S4. Procedural characteristics; Table S5. Bias assessment of the included studies.

Author Contributions: Conceptualization, G.M.; P.G., M.K. and S.S.; methodology, P.G., J.M., M.K. and G.M.; software, validation and formal analysis P.G.; J.M., S.S. and G.M.; data curation, F.A.; J.M. and P.G.; writing-original draft preparation, P.G. and J.M.; writing—review and editing, G.M.; S.S.; M.K.; M.Z.; B.L.; J.S.; S.D.R.; C.I.; J.-P.C.; supervision, G.M. All authors have read and agreed to the published version of the manuscript.

Funding: This research received no external funding

Conflicts of Interest: Dr. Montalescot: reports the following disclosures during the past two years research Grants to the Institution or Consulting/Lecture Fees from ADIR, Amgen, AstraZeneca, Bayer, Berlin Chimie AG, Boehringer Ingelheim, Bristol-Myers Squibb, Beth Israel Deaconess Medical, Brigham Women's Hospital, Cardiovascular Research Foundation, Celladon, CME Resources, Daiichi-Sankyo, Eli-Lilly, Europa, Elsevier, Fédération Française de Cardiologie, Fondazione Anna Maria Sechi per il Cuore, Gilead, ICAN, Janssen, Lead-Up, Menarini, Medtronic, MSD, Pfizer, Sanofi-Aventis, The Medicines Company, TIMI Study Group, WebMD. Dr. Silvain reports consulting fees from Astra-Zeneca, Bayer, Boehringer-Ingelheim, CSL Berhing, Gilead Science; and Sanofi Aventis; Speaker honoraria from AstraZeneca, Amgen, Bayer, Algorythm, and Sanofi-Aventis; and travel support from Amgen, Astra-Zeneca, Bayer, and Bristol-Myer Squibb. Dr. Kerneis has received research grants from Sanofi, Institut Servier and Fédération Française de Cardiologie; consultant fees from Sanofi, Bayer and Servier. Dr. Lattuca has received research grants from Biotronik, Daiichi-Sankyo and Fédération Française de Cardiologie; consultant fees from Daiichi-Sankyo and Eli Lilly; and lecture fees from AstraZeneca and Novartis. Dr. Michel Zeitouni has received research grants from Federation Française de Cardiologie. Institut Servier and Bristol-Myers squibb. Dr. Collet has received research grants or honorarium from AstraZeneca, Bayer, Bristol-Myers Squibb, Daiichi-Sankyo, Eli-Lilly, Fédération Française de Cardiologie, Lead-Up, Medtronic, MSD, Sanofi-Aventis, and WebMD. All other authors do not report any disclosure relative to this study.

\section{References}

1. Yusuf, S.; Zhao, F.; Mehta, S.R.; Chrolavicius, S.; Tognoni, G.; Fox, K.K. Clopidogrel in Unstable Angina to Prevent Recurrent Events Trial Investigators Effects of clopidogrel in addition to aspirin in patients with acute coronary syndromes without ST-segment elevation. N. Engl. J. Med. 2001, 345, 494-502. [PubMed]

2. Levine, G.N.; Bates, E.R.; Bittl, J.A.; Brindis, R.G.; Fihn, S.D.; Fleisher, L.A.; Granger, C.B.; Lange, R.A.; Mack, M.J.; Mauri, L.; et al. 2016 ACC/AHA Guideline Focused Update on Duration of Dual Antiplatelet Therapy in Patients With Coronary Artery Disease: A Report of the American College of Cardiology/American Heart Association Task Force on Clinical Practice Guidelines. J. Am. Coll. Cardiol. 2016, 68, 1082-1115. [CrossRef] [PubMed]

3. Valgimigli, M.; Bueno, H.; Byrne, R.A.; Collet, J.-P.; Costa, F.; Jeppsson, A.; Jüni, P.; Kastrati, A.; Kolh, P.; Mauri, L.; et al. 2017 ESC focused update on dual antiplatelet therapy in coronary artery disease developed in collaboration with EACTS: The Task Force for dual antiplatelet therapy in coronary artery disease of the European Society of Cardiology (ESC) and of the European Association for Cardio-Thoracic Surgery (EACTS). Eur. Heart J. 2018, 39, 213-260. [PubMed]

4. Guedeney, P.; Giustino, G.; Sorrentino, S.; Claessen, B.E.; Camaj, A.; Kalkman, D.N.; Vogel, B.; Sartori, S.; De Rosa, S.; Baber, U.; et al. Efficacy and safety of alirocumab and evolocumab: A systematic review and meta-analysis of randomized controlled trials. Eur. Heart J. 2019. [CrossRef] [PubMed]

5. Palmerini, T.; Benedetto, U.; Biondi-Zoccai, G.; Della Riva, D.; Bacchi-Reggiani, L.; Smits, P.C.; Vlachojannis, G.J.; Jensen, L.O.; Christiansen, E.H.; Berencsi, K.; et al. Long-Term Safety of Drug-Eluting and Bare-Metal Stents: Evidence From a Comprehensive Network Meta-Analysis. J. Am. Coll. Cardiol. 2015, 65, 2496-2507. [CrossRef] [PubMed]

6. Giustino, G.; Baber, U.; Sartori, S.; Mehran, R.; Mastoris, I.; Kini, A.S.; Sharma, S.K.; Pocock, S.J.; Dangas, G.D. Duration of dual antiplatelet therapy after drug-eluting stent implantation: A systematic review and meta-analysis of randomized controlled trials. J. Am. Coll. Cardiol. 2015, 65, 1298-1310. [CrossRef]

7. Armstrong, P.C.J.; Leadbeater, P.D.; Chan, M.V.; Kirkby, N.S.; Jakubowski, J.A.; Mitchell, J.A.; Warner, T.D. In the presence of strong P2Y12 receptor blockade, aspirin provides little additional inhibition of platelet aggregation. J. Thromb. Haemost. 2011, 9, 552-561. [CrossRef]

8. Capodanno, D.; Mehran, R.; Valgimigli, M.; Baber, U.; Windecker, S.; Vranckx, P.; Dangas, G.; Rollini, F.; Kimura, T.; Collet, J.-P.; et al. Aspirin-free strategies in cardiovascular disease and cardioembolic stroke prevention. Nat. Rev. Cardiol. 2018, 15, 480-496. [CrossRef] 
9. Guedeney, P.; Montalescot, G. GLOBAL LEADERS: Looking now at the bigger picture. EuroIntervention 2019, 15, e1030. [CrossRef]

10. Hammoudi, N.; Montalescot, G. The times they are a changin'. Eur. Heart J. 2018, 39, 1736-1739. [CrossRef]

11. Dewilde, W.J.M.; Oirbans, T.; Verheugt, F.W.A.; Kelder, J.C.; De Smet, B.J.G.L.; Herrman, J.-P.; Adriaenssens, T.; Vrolix, M.; Heestermans, A.A.C.M.; Vis, M.M.; et al. Use of clopidogrel with or without aspirin in patients taking oral anticoagulant therapy and undergoing percutaneous coronary intervention: An open-label, randomised, controlled trial. Lancet 2013, 381, 1107-1115. [CrossRef]

12. Gibson, C.M.; Mehran, R.; Bode, C.; Halperin, J.; Verheugt, F.W.; Wildgoose, P.; Birmingham, M.; Ianus, J.; Burton, P.; van Eickels, M.; et al. Prevention of Bleeding in Patients with Atrial Fibrillation Undergoing PCI. N. Engl. J. Med. 2016, 375, 2423-2434. [CrossRef] [PubMed]

13. Cannon, C.P.; Bhatt, D.L.; Oldgren, J.; Lip, G.Y.H.; Ellis, S.G.; Kimura, T.; Maeng, M.; Merkely, B.; Zeymer, U.; Gropper, S.; et al. Dual Antithrombotic Therapy with Dabigatran after PCI in Atrial Fibrillation. N. Engl. J. Med. 2017, 377, 1513-1524. [CrossRef] [PubMed]

14. Vranckx, P.; Valgimigli, M.; Jüni, P.; Hamm, C.; Steg, P.G.; Heg, D.; van Es, G.A.; McFadden, E.P.; Onuma, Y.; van Meijeren, C.; et al. Ticagrelor plus aspirin for 1 month, followed by ticagrelor monotherapy for 23 months vs aspirin plus clopidogrel or ticagrelor for 12 months, followed by aspirin monotherapy for 12 months after implantation of a drug-eluting stent: A multicentre, open-label, randomised superiority trial. Lancet 2018, 392, 940-949. [PubMed]

15. Lopes, R.D.; Heizer, G.; Aronson, R.; Vora, A.N.; Massaro, T.; Mehran, R.; Goodman, S.G.; Windecker, S.; Darius, H.; Li, J.; et al. Antithrombotic Therapy after Acute Coronary Syndrome or PCI in Atrial Fibrillation. N. Engl. J. Med. 2019, 380, 1509-1524. [CrossRef] [PubMed]

16. Watanabe, H.; Domei, T.; Morimoto, T.; Natsuaki, M.; Shiomi, H.; Toyota, T.; Ohya, M.; Suwa, S.; Takagi, K.; Nanasato, M.; et al. Effect of 1-Month Dual Antiplatelet Therapy Followed by Clopidogrel vs 12-Month Dual Antiplatelet Therapy on Cardiovascular and Bleeding Events in Patients Receiving PCI: The STOPDAPT-2 Randomized Clinical Trial. JAMA 2019, 321, 2414-2427. [CrossRef]

17. Hahn, J.-Y.; Song, Y.B.; Oh, J.-H.; Chun, W.J.; Park, Y.H.; Jang, W.J.; Im, E.-S.; Jeong, J.-O.; Cho, B.R.; Oh, S.K.; et al. Effect of P2Y12 Inhibitor Monotherapy vs Dual Antiplatelet Therapy on Cardiovascular Events in Patients Undergoing Percutaneous Coronary Intervention: The SMART-CHOICE Randomized Clinical Trial. JAMA 2019, 321, 2428-2437. [CrossRef]

18. Vranckx, P.; Valgimigli, M.; Eckardt, L.; Tijssen, J.; Lewalter, T.; Gargiulo, G.; Batushkin, V.; Campo, G.; Lysak, Z.; Vakaliuk, I.; et al. Edoxaban-based versus vitamin K antagonist-based antithrombotic regimen after successful coronary stenting in patients with atrial fibrillation (ENTRUST-AF PCI): A randomised, open-label, phase 3b trial. Lancet 2019, 394, 1335-1343. [CrossRef]

19. Mehran, R.; Baber, U.; Sharma, S.K.; Cohen, D.J.; Angiolillo, D.J.; Briguori, C.; Cha, J.Y.; Collier, T.; Dangas, G.; Dudek, D.; et al. Ticagrelor with or without Aspirin in High-Risk Patients after PCI. N. Engl. J. Med. 2019, 381, 2032-2042. [CrossRef]

20. Sorrentino, S.; Giustino, G.; Mehran, R.; Kini, A.S.; Sharma, S.K.; Faggioni, M.; Farhan, S.; Vogel, B.; Indolfi, C.; Dangas, G.D. Everolimus-Eluting Bioresorbable Scaffolds Versus Everolimus-Eluting Metallic Stents. J. Am. Coll. Cardiol. 2017, 69, 3055-3066. [CrossRef]

21. Franzone, A.; McFadden, E.; Leonardi, S.; Piccolo, R.; Vranckx, P.; Serruys, P.W.; Benit, E.; Liebetrau, C.; Janssens, L.; Ferrario, M.; et al. Ticagrelor Alone Versus Dual Antiplatelet Therapy From 1 Month After Drug-Eluting Coronary Stenting. J. Am. Coll. Cardiol. 2019, 74, 2223-2234. [CrossRef] [PubMed]

22. Tomaniak, M.; Storey, R.F.; Serruys, P.W. Aspirin-free antiplatelet regimens after PCI: When is it best to stop aspirin and who could ultimately benefit? EuroIntervention 2019, 15, 1125-1129. [CrossRef]

23. Wiviott, S.D.; Braunwald, E.; McCabe, C.H.; Montalescot, G.; Ruzyllo, W.; Gottlieb, S.; Neumann, F.-J.; Ardissino, D.; De Servi, S.; Murphy, S.A.; et al. Prasugrel versus clopidogrel in patients with acute coronary syndromes. N. Engl. J. Med. 2007, 357, 2001-2015. [CrossRef] [PubMed]

24. Wallentin, L.; Becker, R.C.; Budaj, A.; Cannon, C.P.; Emanuelsson, H.; Held, C.; Horrow, J.; Husted, S.; James, S.; Katus, H.; et al. Ticagrelor versus clopidogrel in patients with acute coronary syndromes. N. Engl. J. Med. 2009, 361, 1045-1057. [CrossRef] [PubMed]

25. Mauri, L.; Kereiakes, D.J.; Yeh, R.W.; Driscoll-Shempp, P.; Cutlip, D.E.; Steg, P.G.; Normand, S.-L.T.; Braunwald, E.; Wiviott, S.D.; Cohen, D.J.; et al. Twelve or 30 months of dual antiplatelet therapy after drug-eluting stents. N. Engl. J. Med. 2014, 371, 2155-2166. [CrossRef] 
26. Généreux, P.; Giustino, G.; Witzenbichler, B.; Weisz, G.; Stuckey, T.D.; Rinaldi, M.J.; Neumann, F.-J.; Metzger, D.C.; Henry, T.D.; Cox, D.A.; et al. Incidence, Predictors, and Impact of Post-Discharge Bleeding After Percutaneous Coronary Intervention. J. Am. Coll. Cardiol. 2015, 66, 1036-1045. [CrossRef]

27. Golwala, H.B.; Cannon, C.P.; Steg, P.G.; Doros, G.; Qamar, A.; Ellis, S.G.; Oldgren, J.; Ten Berg, J.M.; Kimura, T.; Hohnloser, S.H.; et al. Safety and efficacy of dual vs. triple antithrombotic therapy in patients with atrial fibrillation following percutaneous coronary intervention: A systematic review and meta-analysis of randomized clinical trials. Eur. Heart J. 2018, 39, 1726-1735a. [CrossRef]

28. Lopes, R.D.; Hong, H.; Harskamp, R.E.; Bhatt, D.L.; Mehran, R.; Cannon, C.P.; Granger, C.B.; Verheugt, F.W.A.; Li, J.; Ten Berg, J.M.; et al. Safety and Efficacy of Antithrombotic Strategies in Patients With Atrial Fibrillation Undergoing Percutaneous Coronary Intervention: A Network Meta-analysis of Randomized Controlled Trials. JAMA Cardiol. 2019. [CrossRef]

29. Silvain, J.; Cayla, G.; Hulot, J.-S.; Finzi, J.; Kerneis, M.; O'Connor, S.A.; Bellemain-Appaix, A.; Barthélémy, O.; Beygui, F.; Collet, J.-P.; et al. High on-thienopyridine platelet reactivity in elderly coronary patients: The SENIOR-PLATELET study. Eur. Heart J. 2012, 33, 1241-1249. [CrossRef]

30. Cayla, G.; Cuisset, T.; Silvain, J.; Leclercq, F.; Manzo-Silberman, S.; Saint-Etienne, C.; Delarche, N.; Bellemain-Appaix, A.; Range, G.; El Mahmoud, R.; et al. Platelet function monitoring to adjust antiplatelet therapy in elderly patients stented for an acute coronary syndrome (ANTARCTIC): An open-label, blinded-endpoint, randomised controlled superiority trial. Lancet 2016, 388, 2015-2022. [CrossRef]

31. Schüpke, S.; Neumann, F.-J.; Menichelli, M.; Mayer, K.; Bernlochner, I.; Wöhrle, J.; Richardt, G.; Liebetrau, C.; Witzenbichler, B.; Antoniucci, D.; et al. Ticagrelor or Prasugrel in Patients with Acute Coronary Syndromes. N. Engl. J. Med. 2019, 381, 1524-1534. [CrossRef] [PubMed]

32. Collet, J.-P.; Silvain, J.; Montalescot, G. Long-term secondary prevention after high-risk stenting: A good drug for a bad stent. Circulation 2015, 131, 13-16. [CrossRef] [PubMed]

(C) 2020 by the authors. Licensee MDPI, Basel, Switzerland. This article is an open access article distributed under the terms and conditions of the Creative Commons Attribution (CC BY) license (http://creativecommons.org/licenses/by/4.0/). 